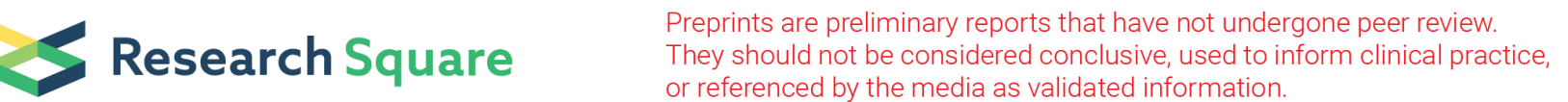

\section{The Proximal Left Anterior Descending Coronary Artery Tortuosity and Angle Associated with Severe Proximal Left Anterior Descending Coronary Artery Stenosis}

\section{Seong Ho Moon}

Gyeongsang National University College of Medicine, Gyeongsang National University Changwon Hospital

\section{Jong Woo Kim}

Gyeongsang National University College of Medicine, Gyeongsang National University Changwon Hospital

\section{Jun Ho Yang}

Gyeongsang National University College of Medicine, Gyeongsang National University Changwon Hospital

\section{Dong Hoon Kang}

Gyeongsang National University College of Medicine, Gyeongsang National University Changwon Hospital

\section{Sung Hwan Kim}

Gyeongsang National University College of Medicine, Gyeongsang National University Changwon Hospital

Jae Jun Jung

Gyeongsang National University College of Medicine, Gyeongsang National University Changwon Hospital

\section{Jong Hwa Ahn}

Gyeongsang National University College of Medicine, Gyeongsang National University Changwon Hospital

\section{Sung Eun Park}

Gyeongsang National University College of Medicine

\section{Kyung Nyeo Jeon}

Gyeongsang National University College of Medicine

\section{Joung Hun Byun ( $\nabla$ jhunikr@naver.com )}

Gyeongsang National University College of Medicine, Gyeongsang National University Changwon Hospital 


\section{Research Article}

Keywords: coronary arteries, coronary artery disease, tortuosity, bifurcation

Posted Date: April 12th, 2021

DOl: https://doi.org/10.21203/rs.3.rs-401420/v1

License: (c) (i) This work is licensed under a Creative Commons Attribution 4.0 International License. Read Full License 


\section{Abstract}

We aimed to describe two factors (coronary artery tortuosity and bifurcation angle) as one descriptor for the evaluation of proximal left anterior descending coronary artery (LAD) disease. We reviewed the medical records of 133 consecutive patients who underwent computed tomography angiography (CTA) for angina symptoms in November 2019 and January 2020. The patients were divided into two groups according to the presence of significant LAD stenosis on CTA (defined as LAD stenosis $>50 \%$ ). The straight length of the vessel was measured using the central luminal line of the flow path, and, calculated using proprietary algorithms in TeraRecon software. We used three-dimensional volume rendering and two-dimensional axial images to measure the left main coronary artery (LM)-LAD angles.

In the univariate analysis, there were significant differences in the linear distance between the endpoints of the $20 \mathrm{~mm}$ actual curve of the LAD (d20), cosine value for LM-LAD angle $(\cos \theta)<0.8$, age, presence of hypertension or diabetes, and number of pack years. However, in the multivariate analysis, the $\cos \theta$ multiplied by the $\mathrm{d} 20\left(\mathrm{~d} 20^{*} \cos \theta\right)<15.5$, presence of hypertension and number of pack years were predictors of significant proximal LAD stenosis. In conclusion, $\mathrm{d} 20 * \cos \theta$ might be useful as a predictor of proximal LAD stenosis.

\section{Introduction}

The coronary artery tortuosity and bifurcation angle are known to affect the vascular wall shear stress and are hemodynamic factors that contribute to the development of atherosclerosis ${ }^{1,2}$. Low shear stress is thought to be atherogenic ${ }^{3}$. Few studies have evaluated coronary artery disease through structural analysis of the proximal left anterior descending coronary artery (LAD). Specifically, there are no studies that have simultaneously considered the roles of the coronary artery tortuosity and bifurcation angle in atherosclerosis. Either of the two factors (coronary artery tortuosity and bifurcation angle) does not independently affect the vessel wall. Both factors affect the vessel wall in combination. Therefore, we aimed to combine these two factors into one descriptor for the evaluation of proximal LAD disease.

\section{Methods}

This retrospective study was approved by Gyeongsang National University Changwon Hospital institutional review board (approval number GNUCH 2020-09-017-002) confirming that all experiments were performed in accordance with relevant guidelines and regulations. Since this study retrospectively collected the existing data, Gyeongsang National University Changwon Hospital institutional review board waived informed consent. The data was recorded in an anonymous manner such that subjects cannot be identified directly or through identifiers linked to the subject. We reviewed the medical records of 133 consecutive patients who underwent computed tomography angiography (CTA) in November 2019 and January 2020. Men and women aged $\geq 20$ years who underwent CTA with angina symptoms between November 2019 and January 2020 were eligible, excluding patients who underwent CTA after previous coronary artery bypass surgery or percutaneous coronary intervention. The patients were divided into two 
groups according to the presence of significant LAD stenosis on CTA (defined as LAD stenosis $>50 \%$ ), as interpreted by radiologists.

The LM-LAD angle ( $\theta$ ) was defined as the angle between the left main coronary artery (LM) and LAD. The coronary tortuosity index (CTI) was defined as the true length of the central luminal line divided by the straight distance. It was calculated from the LAD ostium to $20 \mathrm{~mm}$ away (Figure 1). d20 was defined as the linear distance between the endpoints of the $20-\mathrm{mm}$ actual curve of the $L A D$, and $d 20 * \cos \theta$ was defined as $\cos \theta$ multiplied by $\mathrm{d} 20$.

Analysis and measurement of the coronary computed tomographic angiography (cCTA) scans were performed using commercially available post-processing software linked to the institutional picture archiving and communication system (PACS; version 4.4.11; Aquarius iNtuition TeraRecon, Foster City, CA, USA). Computed tomography (CT) scans were loaded and an independent review of the imaging data for each patient was performed by one investigator who received instructions on the standardized methods for the measurement of the degree of stenosis in the LAD and coronary tortuosity index (CTI) of the proximal LAD. The CTI was defined as the true length of the central luminal line divided by the straight distance. It was calculated from LAD ostium to $20 \mathrm{~mm}$ away (Fig.1). The straight length of the vessel was measured using the central luminal line of the flow path and was calculated using proprietary algorithms in TeraRecon software (TeraRecon, Foster City, CA, USA). Three-dimensional volume rendering and two-dimensional axial images were used to measure the angles between the left main coronary artery (LM) and LAD (Fig. 2).

All cCTAs were performed using a third generation dual-source CT scanner (Siemens SOMATOM Force, Siemens Healthineers, Forchheim, Germany). At heart rates $>60$ bpm, intravenous beta blockers were administered prior to cCTA and, in the absence of contraindications, sublingual nitroglycerin $(0.8 \mathrm{mg})$ was applied at all times. lodinated contrast material $(200 \mathrm{~mL}$; Iomeron 400; Bracco Imaging S.p.A., Milan, Italy) was injected using a power injector at a flow rate of $5 \mathrm{~mL} / \mathrm{s}$, followed by a $50 \mathrm{~mL}$ saline chaser.

\section{Statistical Methods}

Continuous variables were tested for normal distribution using the Kolmogorov-Smirnov test. In cases of normal distribution, continuous variables are presented as mean \pm standard deviation and were compared using Student's t-test. The Chi-square test or Fisher's exact test was used to compare categorical variables between groups. Binary logistic regression analysis was used to determine the independent predictors of predictors of significant proximal LAD stenosis. The odds ratio (OR) and its $95 \%$ confidence interval $(\mathrm{Cl})$ were calculated. A receiver-operating characteristic $(\mathrm{ROC})$ curve analyses of $\mathrm{d} 20, \cos \theta$, and $\mathrm{d} 20^{*} \cos \theta$ were performed to identify the optimal cutoff value for the prediction of significant proximal LAD stenosis. The optimal cutoff point of CACS was calculated by the Youden $\mathrm{J}$ statistic $^{4}$. Statistical analysis was performed using SPSS for Windows, version 21 (IBM Corporation, Chicago, IL). A two-tailed P-value $<0.05$ was considered significant. 


\section{Results And Discussion}

Baseline patient characteristics are shown in Table 1. Proximal LAD stenosis of $>50 \%$ was seen in 26 of the 133 patients. The two groups differed significantly in the age, presence of hypertension or, diabetes, and number of pack years $(P=0.017, P=0.032, P=0.034$, and $P=0.011$, respectively); however, there were no other significant differences. The mean LM-LAD angles of patients with $<50 \%$ and $>50 \%$ proximal LAD stenosis were $29.2^{\circ}$ and $38.3^{\circ}$, respectively. Table 2 shows that the LM-LAD angles and $\cos \theta$ differed significantly between the two groups ( $P<0.001$ for both). There was no statistically significant difference in the linear distances between the $d 20$ endpoints between the two groups $(P=$ $0.104)$; however, there was a statistically significant difference in $d 20 * \cos \theta(P<0.001)$. Among the patients with $>50 \%$ proximal LAD stenosis, $65.4 \%$ and $73.1 \%$ of patients had a d $20<19 \mathrm{~mm}$ and $\cos \theta<$ 0.8 , respectively. The areas under the ROC curves for $\mathrm{d} 20, \cos \theta$ and $\mathrm{d} 20 * \cos \theta$ were $0.634,0.743$ and 0.767 , respectively. The cut-off values of $19 \mathrm{~mm}, 0.8$, and 15.5 for $\mathrm{d} 20, \cos \theta$, and $\mathrm{d} 20^{*} \cos \theta$, respectively, had sensitivities and specificities of 65.4 and 58.5, 73.1 and 74.8, and 80.8 and 71, respectively (Fig. 3). In the univariate analysis (Table 3), there were significant differences in the $d 20, \cos \theta<0.8$, age, presence of hypertension or diabetes, and number of pack years (hazard ratio [HR]: 2.70, 8.04, 1.05, 3.70, 2.82, and 1.04; 95\% Cl: 1.13-6.87, 3.17-22.57, 1.01-1.09, 1.30-13.30, 1.16-6.88, and 1.01-1.06; $P=0.029, \mathrm{P}<$ $0.001, P=0.020, P=0.024, P=0.021$, and $P=0.002$, respectively). However, in the multivariate analysis (Table 4), the d20* $\cos \theta<15.5$, presence of hypertension, and number of pack years (HR: $11.36,4.54$, and 1.04; 95\% Cl: 3.9-39.54, 1.39-18.32, and 1.01-1.07; and $P<0.001, P=0.019$, and $P=0.003$, respectively) were predictors of significant proximal LAD stenosis. 
Table 1

Baseline characteristics between the two groups $(n=133)$

LAD stenosis $<50 \%$

LAD stenosis $\geq 50 \%$

P-value

$(n=107) \quad(n=26)$

\begin{tabular}{llll} 
Age (year) & $59.7 \pm 12.8$ & $66.3 \pm 10.8$ & 0.017 \\
\hline Male $(\%)$ & $58(54.2)$ & $18(69.2)$ & 0.243 \\
\hline Height $(\mathrm{cm})$ & $163.3 \pm 9.2$ & $163.8 \pm 7.8$ & 0.794 \\
\hline Body mass index & $24.6 \pm 4.0$ & $24.5 \pm 6.3$ & 0.948 \\
$(\mathrm{~kg} / \mathrm{m} 2)$ & & $22(84.6)$ & 0.032 \\
\hline Hypertension (\%) & $64(59.8)$ & $18(69.2)$ & 0.821 \\
\hline Dyslipidemia (\%) & $69(64.5)$ & $2(7.7)$ & 0.730 \\
\hline Chronic kidney disease $(\%)$ & $4(3.7)$ & $13(50.0)$ & 0.034 \\
\hline Diabetes $(\%)$ & $28(26.2)$ & $20.4 \pm 22.8$ & 0.011 \\
\hline Pack years & $7.6 \pm 14.2$ & $3(11.5)$ & 0.651
\end{tabular}

Data are presented as mean \pm standard deviation or number (percentage). LAD, left anterior descending artery

Table 2

Calculated figures and parameters measured by computed tomography angiography

\begin{tabular}{|c|c|c|c|}
\hline & LAD stenosis $<50 \%$ & LAD stenosis $\geq 50 \%$ & P-value \\
\hline & $(n=107)$ & $(n=26)$ & \\
\hline LM-LAD $\left(^{\circ}\right)$ & $29.2 \pm 10.9$ & $38.3 \pm 8.7$ & $<0.001$ \\
\hline $\cos \theta$ & $0.9 \pm 0.1$ & $0.8 \pm 0.1$ & $<0.001$ \\
\hline d20 & $19.0 \pm 0.8$ & $18.7 \pm 0.7$ & 0.104 \\
\hline $\mathrm{d} 20^{*} \cos \theta$ & $16.3 \pm 2.0$ & $14.5 \pm 1.8$ & $<0.001$ \\
\hline $\mathrm{d} 20<19 \mathrm{~mm}(\%)$ & $44(41.1)$ & $17(65.4)$ & 0.045 \\
\hline $\cos \theta<0.8(\%)$ & $27(25.2)$ & $19(73.1)$ & $<0.001$ \\
\hline
\end{tabular}


Table 3

Results of the univariate logistic regression analyses for predictors of significant proximal LAD stenosis.

\section{Univariate $\mathrm{OR}(95 \% \mathrm{Cl})$}

$\mathrm{d} 20<19 \mathrm{~mm}$

$\cos \theta<0.8$

Age

Male

Body mass index

Hypertension

Dyslipidemia

Chronic kidney disease

Diabetes

Pack year

Family history

Data are presented as mean \pm standard deviation or number (percentage). OR, odds ratio; $\mathrm{Cl}$, confidence interval; d20, linear distance between the endpoints of the $20 \mathrm{~mm}$ actual curve of the LAD; $\cos \theta, \operatorname{cosine}$ value for LM-LAD angle

Table 4

Results of the multivariate logistic regression analyses for predictors of significant proximal LAD stenosis.

\begin{tabular}{|lll|}
\hline & Multivariate OR (95\% Cl) & P-value \\
\hline $\mathrm{d} 20 * \cos \theta<15.5$ & $11.36(3.9-39.54)$ & $<0.001$ \\
\hline Hypertension & $4.54(1.39-18.32)$ & 0.019 \\
\hline Pack year & $1.04(1.01-1.07)$ & 0.003 \\
\hline $\begin{array}{l}\text { Data are presented as mean } \pm \text { standard deviation or number (percentage). OR, odds ratio; } \mathrm{Cl} \\
\text { confidence interval; } \text { d20, the linear distance between the endpoints of the 20mm actual curve of the } \\
\text { LAD; } \cos \theta, \text { cosine value for LM-LAD angle }\end{array}$ \\
\hline
\end{tabular}

In the present report, we provided the importance of the combined factors $(\mathrm{d} 20 * \cos \theta)$ of the LM-LAD angle and the LAD tortuosity when considering risk or predicting factors of significant proximal LAD stenosis. That means that the structural analysis of the patient's coronary artery is necessary in the analysis for the cause of coronary artery disease, and is also important for patient management. 
Atherosclerosis is a chronic disease that results in insufficient blood supply to the heart muscle which can lead to angina or heart attacks ${ }^{1}$. Metabolic problems are considered causative factors for atherosclerosis of blood vessels; however, there are structural factors that are also considered important contributors to this disease. Coronary bifurcation angles influence plaque initiation in the coronary artery $^{2}$.

We previously performed a study which found that the LM-LAD angle was, statistically, a more significant factor in the formation of the LAD stenotic lesions than the LAD-left circumflex artery (LCX) angle ${ }^{3}$. Malvè et al. reported that the LM-LAD angle is a better predictor of low shear stress than the LM-LCX angle ${ }^{5}$. In addition, coronary artery tortuosity leads to a variation in blood flow and affects the local wall shear stress $^{6}$. The tortuosity index $(T I)$ is the distance factor defined as $T I=(L / D)-1$ : where $L$ is the vessel length, and $D$ is the straight line distance between its end point ${ }^{7}$.

Both the coronary artery bifurcation angles and tortuosity change the shear stress in blood vessels. In general, low shear stress is a causative factor for atherosclerosis ${ }^{8-11}$. To our knowledge, there has been little research into the structural analysis of proximal LAD lesions. In particular, few studies have simultaneously analyzed the tortuosity and bifurcation angle.

The wall shear stress $(\tau)$ can be expressed as $\tau=4 \eta Q / \pi r 3$,

where $\eta$ is the apparent blood viscosity ( 0.035 Poise), $Q$ is the rate of blood flow through the vessel, and $r$ is the vessel radius. The shear stress increases when the blood flow increases or blood vessel diameter decreases, and the shear stress decreases as the blood flow decreases ${ }^{12}$.

A wider coronary artery bifurcation angles, regions of low shear stress form in the areas of bifurcation ${ }^{13}$, 14 , and the fluid shear stress gradients (flow acceleration and deceleration) that are created in tortuous vessels have been shown to initiate platelet aggravation ${ }^{15}$.

The proximal LAD forms a bifurcation angle, branches at the LM, and has tortuosity. Its blood flow is influenced by two factors, bifurcation angle and tortuosity. In other words, as the proximal LAD bifurcation angle increases, regions of low shear stress occur, Subsequently, regions of high and low shear stress occur on the bent outer and inner sides, respectively, resulting in shear gradients.

Using this rationale, we described the bifurcation angle and tortuosity as one $(\mathrm{d} 20 * \cos \theta)$. The vessel length was fixed at $20 \mathrm{~mm}$, thus the $\mathrm{d} 20$ was the only variable that was used to represent tortuosity. This equation did not come from a physical or mathematical calculation. Both factors have close effects on the shear stress, and the resulting effects on the vessel could also be considered closely related. Thus, it was expressed as a product of two factors and the results were statistically significant (Table 4 ). In particular, when $\mathrm{d} 20 * \cos \theta<15.5$, the risk of proximal LAD lesion formation was high. To satisfy the inequality of $d 20^{*} \cos \theta<15.5, \mathrm{~d} 20$ should be $<19.94$ when $\theta=39^{\circ}$. However, when $\theta>40^{\circ}$, d20 should be $<20.23$. Since $\mathrm{d} 20$ is always $<20$, all d20 values always satisfy the inequality at angles above $40^{\circ}$. 
When $\mathrm{d} 20$ is 20 , that is, when there is no bending, the LM-LAD angle must be $>39^{\circ}$ to satisfy the inequality. In summary, when there is no bending, the risk of proximal LAD lesion formation occurs at an $L M-L A D$ angle $>39^{\circ}$. In other words, if the LM-LAD angle is $<39^{\circ}$, the risk of proximal LAD lesion formation is affected by both the $d 20$ and the LM-LAD angle. If it is $>40^{\circ}$, the risk of proximal LAD lesions is affected by the angle rather than the tortuosity. And in the absence of tortuosity of LAD, risk of proximal LAD lesions are affected when the LM-LAD angle is $>40^{\circ}$. In the study that we performed previously ${ }^{3}$, we found that an $L M-L A D$ angle $>40^{\circ}$ was a predictor for significant LAD stenosis.

This was a single center, retrospective study, and the sample size of the number of patients with significant LAD stenosis was small. A larger, multi-center, prospective study is needed. Since the length of the proximal LAD was measured up to $20 \mathrm{~mm}$, it may be difficult to apply our findings to the entire area of the coronary artery.

The findings of this study are too complicated to generalize, because the entire LAD does not only bend once and there are several changes in the blood vessel route. In our next study, we plan to repeat our measurements and include measurements that are divided into the mid and, distal LAD to express a generalizable formula that may be applicable to the entire LAD.

\section{Conclusion}

As the tortuosity increased and the LM-LAD angle increased ( $\mathrm{d} 20$ and $\cos \theta$ decreased, respectively), the shear stress gradient on the inner wall of the vessel increased, which increased the chance of proximal LAD lesion formation. Since $d 2{ }^{*} \cos \theta$ can be easily measured clinically, it might be useful as a predictor of proximal LAD stenosis.

\section{Declarations}

\section{Data availability}

The data used and analyzed in this work are available from the corresponding author upon reasonable request.

This date is provided to supplementary information.

\section{Acknowledgements}

The authors are grateful to Byeong Yeol Ahn and Tae Hee Kim from Changwon Science High School for their physical and mathematical opinions.

\section{Sources of Funding}

None 


\section{Contributions}

B.J.H.: conceptualization, methodology, formal analysis, investigation, writing: original draft, supervision M.S.H.: investigation, writing: original draft, visualization. K.J.W.: data curation. Y.J.H.: methodology, validation. K.D.H.: editing. K.S.H.: methodology. J.J.J : investigation. A.J.H : methodology, visualization. P.S.E : methodology, visualization. J.K.N : methodology, visualization.

\section{Corresponding author}

Correspondence to Joung Hun Byun

\section{Additional information}

I declare that the authors have no competing interests as defined by Nature Research, or other interests that might be perceived to influence the results and/or discussion reported in this paper.

\section{References}

1. Buradi A, Mahalingam A. Impact of coronary tortuosity on the artery hemodynamics. Biocybern Biomed Eng. 2020;40:126-147. doi: 10.1016/j.bbe.2019.02.005.

2. Morbiducci U, Kok AM, Kwak BR, Stone PH, Steinman DA, Wentzel JJ. atherosclerosis at arterial bifurcations: evidence for the role of haemodynamics and geometry. Thromb Haemost. 2016;115:484-492. doi: 10.1160/th15-07-0597.

3. Moon SH, Byun JH, Kim JW, Kim SH, Kim KN, Jung JJ, et al. Clinical usefulness of the angle between left main coronary artery and left anterior descending coronary artery for the evaluation of obstructive coronary artery disease. PloS one. 2018;13:e0202249. doi: 10.1371/journal.pone.0202249.

4. Hajian-Tilaki K. Receiver operating characteristic (ROC) curve analysis for medical diagnostic test evaluation. Caspian J Intern Med. 2013;4:627-635.

5. Malvè M, Gharib AM, Yazdani SK, Finet G, Martínez MA, Pettigrew R, et al. Tortuosity of coronary bifurcation as a potential local risk factor for atherosclerosis: CFD steady state study based on in vivo dynamic CT measurements. Ann Biomed Eng. 2015;43:82-93. doi: 10.1007/s10439-014-1056-y.

6. Li Y, Liu NF, Gu ZZ, Chen Y, Lu J, Feng Y, et al. Coronary tortuosity is associated with reversible myocardial perfusion defects in patients without coronary artery disease. Chin Med J (Engl). 2012;125:3581-3583. doi: 10.3760/cma.j.issn.0366-6999.2012.19.041.

7. Dougherty G, Varro J. A quantitative index for the measurement of the tortuosity of blood vessels. Med Eng Phys. 2000;22:567-574. doi: 10.1016/s1350-4533(00)00074-6.

8. Malek AM, Alper SL, Izumo S. Hemodynamic shear stress and its role in atherosclerosis. JAMA. 1999;282:2035-2042. doi: 10.1001/jama.282.21.2035. 
9. Han SH, Puma J, Garcia-Garcia HM, Nasu K, Margolis P, Leon MB, et al. Tissue characterisation of atherosclerotic plaque in coronary artery bifurcations: an intravascular ultrasound radiofrequency data analysis in humans. Eurolntervention. 2010;6:313-320. doi: 10.4244/eijv6i3a53.

10. Gijsen FJ, Wentzel JJ, Thury A, Lamers B, Schuurbiers JC, Serruys PW, et al. a new imaging technique to study 3-D plaque and shear stress distribution in human coronary artery bifurcations in vivo. $\mathrm{J}$ Biomech. 2007;40:2349-2357. doi: 10.1016/j.jbiomech.2006.12.007.

11. Cecchi E, Giglioli C, Valente S, Lazzeri C, Gensini GF, Abbate R, et al. Role of hemodynamic shear stress in cardiovascular disease. Atherosclerosis. 2011;214:249-256. doi: 10.1016/j.atherosclerosis.2010.09.008.

12. Behnke BJ, Prisby RD, Lesniewski LA, Donato AJ, Olin HM, Delp MD. Influence of ageing and physical activity on vascular morphology in rat skeletal muscle. J Physiol. 2006;575:617-626. doi: 10.1113/jphysiol.2006.108431.

13. Juan YH, Tsay PK, Shen WC, Yeh CS, Wen MS, Wan YL. Comparison of the left main coronary bifurcating angle among patients with normal, non-significantly and significantly stenosed left coronary arteries. Sci Rep. 2017;7:1515. doi: 10.1038/s41598-017-01679-3.

14. Sun Z, Cao Y. Multislice ct angiography assessment of left coronary artery: correlation between bifurcation angle and dimensions and development of coronary artery disease. Eur J Radiol. 2011;79:e90-e95. doi: 10.1016/j.ejrad.2011.04.015.

15. Luna DJ, Pandian NK, Mathur T, Bui J, Gadangi P, Kostousov VV, et al Tortuosity-powered microfluidic device for assessment of thrombosis and antithrombotic therapy in whole blood. Sci Rep. 2020;10:5742. doi: 10.1038/s41598-020-62768-4.

\section{Figures}




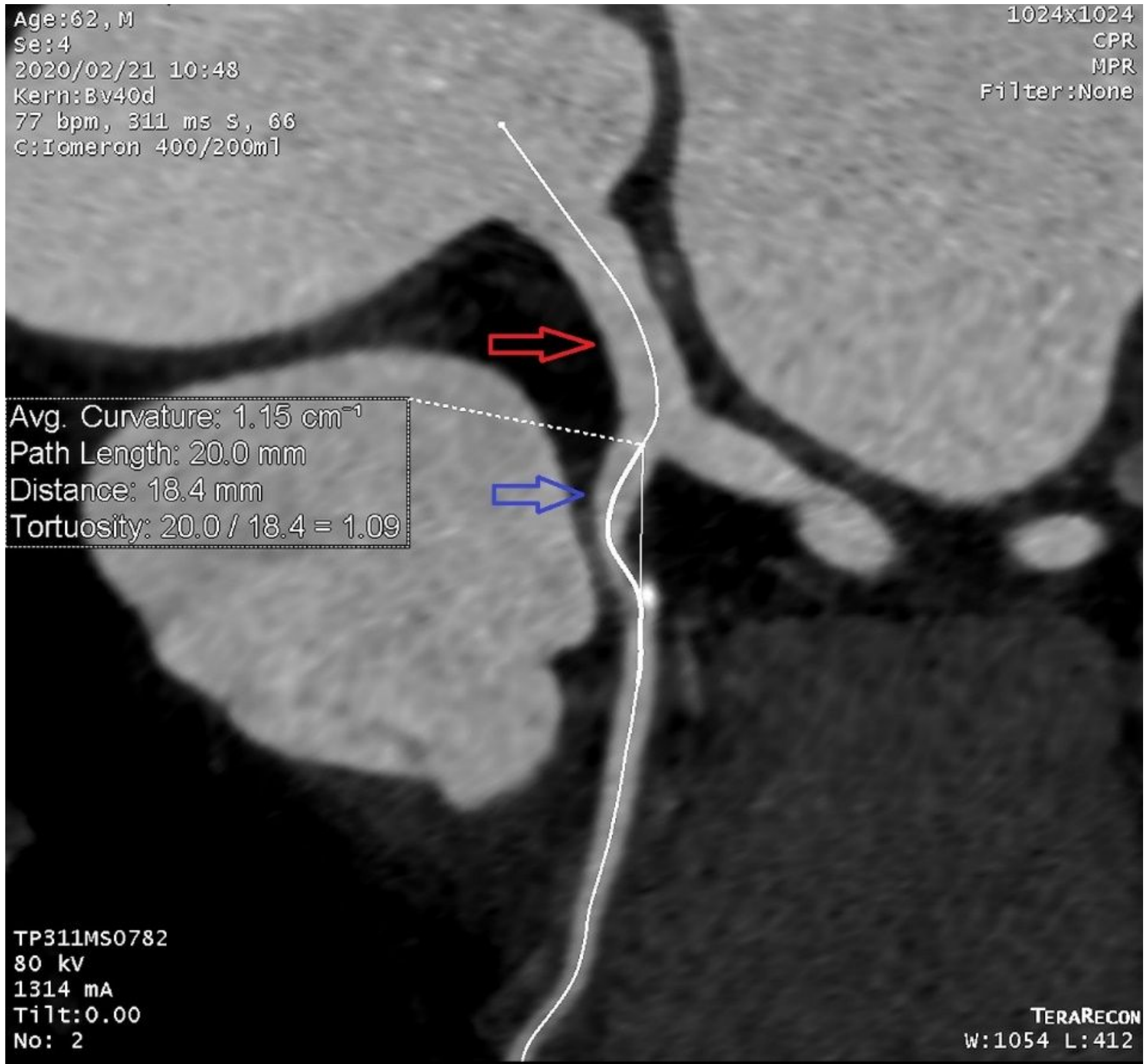

\section{Figure 1}

A representative image of the measurement of the linear distance between the endpoints of the $20-\mathrm{mm}$ actual curve of the left anterior descending coronary artery (LAD) on computed tomography angiography. The linear distance between the endpoints of the $20 \mathrm{~mm}$ actual curve of the LAD (blue arrow) is $18.4 \mathrm{~mm}$. The red arrow is the left main coronary artery. 


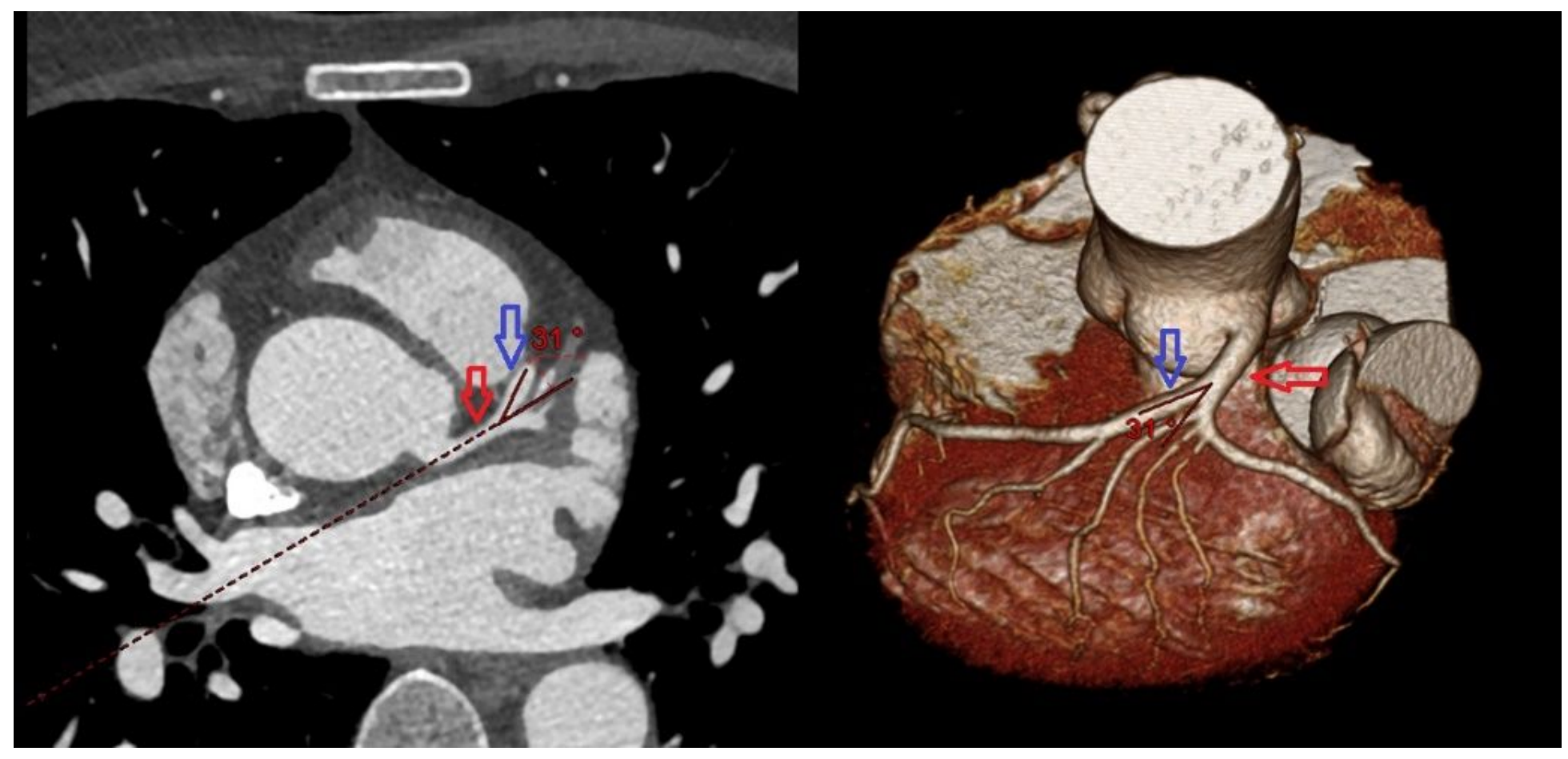

Figure 2

Measurement of an angle on computed tomography angiography. The angle between the left main coronary artery (red arrow) and left anterior descending coronary artery (blue arrow) is $31^{\circ}$. 


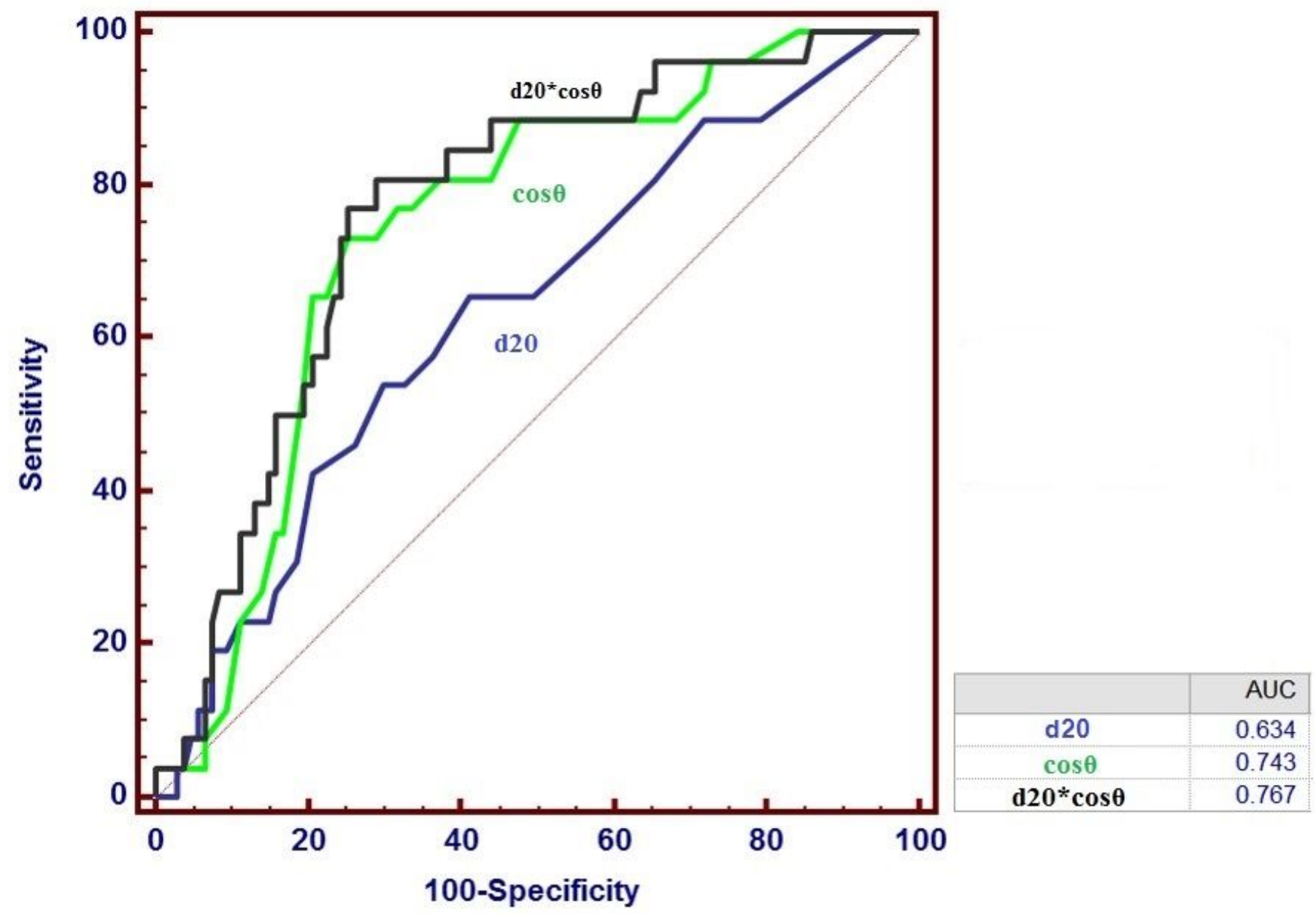

Figure 3

The receiver operating characteristic analysis to establish whether $d 20, \cos \theta$ and $d 20^{\star} \cos \theta$ signify significant proximal LAD stenosis. AUC, area under the curve.

\section{Supplementary Files}

This is a list of supplementary files associated with this preprint. Click to download.

- data.xlsx 